Revue d'histoire de l'Amérique française

REVUE D.HISTOIRE DE L'AMÉRIQUE FRANÇAISE

\title{
Artilleurs canadiens-français dans la bataille de Normandie (juillet-août 1944) (suite)
}

\section{Jacques Gouin}

Volume 16, numéro 4, mars 1963

URI : https://id.erudit.org/iderudit/302229ar

DOI : https://doi.org/10.7202/302229ar

Aller au sommaire du numéro

Éditeur(s)

Institut d'histoire de l'Amérique française

ISSN

0035-2357 (imprimé)

1492-1383 (numérique)

Découvrir la revue

Citer cet article

Gouin, J. (1963). Artilleurs canadiens-français dans la bataille de Normandie (juillet-août 1944) (suite). Revue d'histoire de l'Amérique française, 16(4), 536-548. https://doi.org/10.7202/302229ar d'utilisation que vous pouvez consulter en ligne.

https://apropos.erudit.org/fr/usagers/politique-dutilisation/ 


\section{ARTILLEURS CANADIENS-FRANÇAIS DANS LA BATAILLE DE NORMANDIE * \\ (juillet-août 1944) \\ (suite)}

3e article

\section{CHEVAUCHÉE POLONAISE, DE VAUCELLES À CHAMBOIS}

\section{HARDIESSE DES POLONAIS}

La période qui suivit le départ de Vaucelles, le 8 août, fut pour le Régiment une première initiation à la guerre de mouvements. En effet, dès le lendemain de son installation à HubertFolie, le Régiment recevait ordre de la $1^{\text {re }}$ division polonaise d'établir une nouvelle position à Crâménil, quelques milles plus loin ${ }^{1}$. Mais, dans leur impatience à détruire les Allemands, les Polonais étaient portés plus que de raison, semble-t-il, à s'aventurer un peu trop loin. Leur hardiesse s'explique d'ailleurs facilement, si l'on songe au malheureux martyre de la Pologne. Tous les combattants de la $1^{\text {re }}$ division blindée polonaise, dont le général Maczek le premier, qui avait commandé, en qualité de colonel, une brigade blindée pendant la campagne de Pologne ${ }^{2}$, avaient connu, d'une façon ou d'une autre, le traitement implacable et infamant infligé à leur pays, dès son occupation en octobre 1939 par les troupes SS du sinistre Himmler. Aussi, est-il facile de comprendre l'impatience et l'esprit de revanche qui animaient tous les hommes du général Maczek. D'autre part, pour des raisons également évidentes, les artilleurs du Régiment n'éprouvaient que difficilement pareils sentiments à l'égard des Allemands. Certes, comme tous les autres soldats

* Voir notre Revue, XVI: 24.0-253, 353-368.

1 Journal de guerre du Régiment, XXIX: 5.

2 Robert Jars, La campagne de Pologne (septembre 1939), (Paris, Payot, 1949), 201. 
canadiens, ceux du Régiment étaient venus en Europe pour participer en quelque sorte à une croisade contre le nouvel ordre hitlérien, mais sans éprouver de haine personnelle à l'égard des Allemands. Mais, une fois soumis au commandement des Polonais, il fallait bien qu'ils obéissent aux ordres.

\section{DES OFFICIERS DU REGIMENT, PRIS POUR DES ESPIONS ALLEMANDS, SONT FAITS PRISONNIERS PAR LES POLONAIS}

Or, comme nous l'avons dit plus haut, dès le 9 août, le haut commandement polonais avait ordonné au Régiment d'aller établir une nouvelle position à Crâménil. Le lendemain, il lui ordonnait d'en occuper une autre, encore plus loin, près de Cauvicourt. Une reconnaissance rapide de cette dernière position assignée au Régiment par les Polonais, effectuée comme d'habitude par le major Archer, accompagné d'un officier de chaque troupe et de l'officier topographe, - en l'occurrence, le lieut. Gouin, qui assumait depuis la veille ses nouvelles fonctions, démontra aussitôt que l'endroit était intenable ${ }^{3}$. En effet, dès leur arrivée près de la position assignée, les officiers chargés de la reconnaissance se rendirent compte que l'atmosphère générale avait quelque chose d'étrange: des cadavres allemands gisaient encore en plein soleil, et des fantassins polonais, blottis dans de profondes tranchées, semblaient attendre de pied ferme, baïonnette au canon, l'irruption imminente d'une attaque allemande. Soudain, la haute et mince silhouette du lieutenant Guillaume Geoffrion ${ }^{4}$, de la $50^{\mathrm{e}}$ batterie, émergea du sommet d'une crête. Il avait les mains levées et était poursuivi par un zélé fantassin polonais, à la pointe d'une mitraillette. Ce qui s'était passé, c'est que la reconnaissance s'étant effectuée en territoire allemand avancé, les Polonais avaient pris le groupe de reconnaissance du Régiment pour une poignée d'espions allemands déguisés en Canadiens, et s'apprêtaient à les traiter en conséquence. Il est fort probable que ce qui épargna la vie à ces Canadiens, considérés comme espions, c'est qu'au moment précis où le lieutenant Geoffrion, - toujours flegmatique, malgré

\footnotetext{
3 Journal de guerre du Régiment, XXIX: 6 .
4 Avocat, a repris l'exercice de sa profession à Montréal, après la guerre.
} 
les circonstances, - exhortait le lieutenant Gouin à lever également les mains, sous la menace de la mitraillette du Polonais, les mortiers allemands déclenchèrent un violent tir concentré qui força tous les intéressés, - Polonais et Canadiens compris, à se précipiter aussitôt dans le premier trou disponible. Comme le tir des mortiers allemands ne ralentissait pas, le major Archer, blotti derrière une petite colline, crut bon de faire part de la situation au commandant, par radio. Le commandant décida alors de ne pas faire occuper la position, au grand désappointement des Polonais. Mais il était impossible d'agir autrement sans compromettre la sécurité de tout le Régiment. Tel fut le prélude à la chevauchée polonaise ${ }^{5}$ !

\section{BATAILLE DU BOIS DE QUESNAY}

A ce moment, le point névralgique du front qui faisait face aux Polonais était le bois de Quesnay, «littéralement truffé d'artillerie allemande», selon le témoignage d'un Normand ${ }^{6}$. Aussi, le major Tremblay, toujours de l'avant avec les Polonais, ne cessait de réclamer le feu du Régiment contre des cibles concentrées dans ce bois. Jusque-là, le Régiment avait tiré 300 obus pour les Polonais, avec les huit canons qu'il lui restait, depuis le bombardement du 8 août ${ }^{7}$. Depuis le 13 juillet, il en avait tiré $28,419^{8}$.

Le 11 août, le major Tremblay et le capitaine Chabot étaient relevés de leurs fonctions auprès des Polonais par le major Lahaie et le capitaine Mercier ${ }^{9}$. Le major Tremblay et le capitaine Chabot s'étant acquittés de leur tâche difficile avec éclat, le commandant leur accordait aussitôt un congé bien mérité de trois jours. Déjà, la débâcle allemande commençait à se manifester de façon évidente. "Les prisonniers encombrent nos routes et nos canons accumulent des tas de ferraille...», écrivait un officier du Régiment, le 11 août ${ }^{10}$.

\footnotetext{
Geoffrion.

5 Récit reconstitué et rappelé à l'auteur par le lieutenant Guillaume

6 Herval, op. cit., 384.

7 Journal de guerre du Régiment, XXIX: 6.

8 Ibid.

9 Ibid.

10 Archives de l'auteur, lettre du 11 août 1944.
} 
Le lendemain, le brigadier Suttie, commandant du $2^{\mathrm{e}}$ groupe, rendait visite au Régiment. D'après lui, le Régiment devait rester dans cette même position pour plusieurs jours: mais il ne connaissait pas les Polonais ! En effet, dès le 14 août, le major Archer se rendait de nouveau près de Cauvicourt pour préparer une nouvelle position, à la demande des Polonais ${ }^{11}$. Or, ce jour-là devait se révéler extrêmement mouvementé. D'abord, du côté des Allemands, voici ce qui se passait, d'après le récit oculaire d'un Normand:

L'épisode le plus dramatique de cette journée est celui de la bataille du bois de Quesnay... Cerné par les blindés ... [il] est finalement nettoyé dans la journée après un corps à corps sanglant, au cours duquel plus de mille prisonniers allemands sont capturés... Le chaos de ce bois .... l'un des plus meurtriers de la plaine de Caen, atteste la violence des combats que livrèrent aux Nazis les valeureuses légions de Montgomery ${ }^{12}$.

Du côté canadien et polonais, il faut avouer que, pendant un certain temps, la situation n'était guère plus enviable. En effet, pendant que le groupe de reconnaissance du Régiment arpentait les environs de Cauvicourt, à la recherche d'une position propice à la mise en batterie des canons, vers $2 \mathrm{~h} .30$ de l'après-midi, la catastrophe du 8 août menaçait de s'abattre de nouveau sur le Régiment. En effet, l'aviation britannique cette fois lâchait ses bombes, par erreur, sur l'ensemble du secteur occupé par le Régiment. Heureusement, le Régiment ne perdit qu'un homme, le sergent Pelletier de la $58^{\mathrm{e}}$ batterie. Mais, d'autre part, le Q.G. du $2^{\mathrm{e}}$ groupe et le $7^{\mathrm{e}}$ régiment d'artillerie moyenne subissaient de lourdes pertes. Comme c'était la deuxième fois que pareil bombardement massif s'abattait sur les lignes du Régiment, et cela de la part des avions alliés, le moral du Régiment en fut momentanément ébranlé. On pouvait voir des canonniers courir dans toutes les directions et crier à pleins poumons, littéralement affolés ${ }^{13}$.

11 Journal de guerre du Régiment, XXIX: 8.

12 Herval, op. cit., 385.

13 Journal de guerre du Régiment, XXIX : 8. 


\section{LES ALLEMANDS EN RETRAITE}

Dès 6 heures du soir, malgré ce bombardement aérien dévastateur, le Régiment était en batterie autour de Cauvicourt. Juste en face du Q.G. régimentaire était aménagée une cage de prisonniers allemands où se trouvaient 1500 débris de la Wehrmacht, à bout de souffle. Pendant que la prévôté canadienne s'occupait à briser l'arrogance des officiers SS capturés, le capitaine Brosseau, médecin du Régiment, installé tant bien que mal dans une cave, travaillait sans relâche à panser les plaies béantes que les canons du Régiment avaient infligées chez les Allemands. Ces derniers, en retraite vers l'Est depuis le 13 août, avaient le moral fort entamé. Voici ce qu'écrit à propos de cette période un historien français:

La retraite, d'abord ordonnée, se changea vite en déroute dans un chaos d'hommes et de matériel invraisemblable sur des routes encombrées, tandis que l'aviation pilonnait sans cesse et que l'artillerie tendait un réseau d'obus puissant. Ce fut une tempête de feu terrifiante et un massacre ${ }^{14}$.

Même les officiers allemands «comprenaient qu'ils avaient perdu la partie dans l'Ouest et que les événements de Normandie sonnaient le glas de l'Allemagne nazie ${ }^{15} »$. De fait, dès l'échec de l'offensive de Mortain, le général von Kluge était relevé de son commandement et remplacé par le maréchal Model, appelé exprès de Russie; von Kluge devait se suicider quelques jours plus tard, en route vers l'Allemagne ${ }^{16}$. Dans une lettre, datée du 18 août, von Kluge faisait part à Hitler de son intention de se suicider, mais prenait en même temps la peine de lui expliquer longuement le motif de son geste. Voici quelques extraits révélateurs de cette longue lettre:

Permettez-moi, mon Führer, avec tout le respect que je vous dois, d'exposer mon point de vue. Quand vous recevrez ces lignes... je ne serai plus. Je ne peux supporter le reproche d'avoir scellé le sort de l'Ouest par une stratégie fautive... Prenez la

\footnotetext{
${ }^{14}$ J. Joubert, La libération de la France (Paris, Payot, 1951), 138. 
résolution de mettre fin à la guerre. Le peuple allemand a supporté des souffrances si inouies qu'il est temps de mettre fin à ces choses terribles... Vous avez mené une lutte grande et honorable. L'histoire le prouvera. Montrez que vous êtes grand aujourd'hui pour mettre fin à une lutte sans espoir.... ${ }^{17}$.

Pendant que s'opéraient ces changements importants dans le haut commandement allemand, le Régiment n'en continuait pas moins à harceler implacablement la Wehrmacht en pleine retraite. «La nuit du 15 août est effrayante... Les canons crachent de tous côtés, tandis que les Allemands, désemparés, se replient en direction... de Trun», écrivait plus tard un témoin normand ${ }^{18}$.

A l'aube de ce même jour, 15 août, un nouveau débarquement allié s'effectuait près de Toulon et de Cannes dans le midi de la France. L'après-midi même de ce jour, le Régiment avançait vers une nouvelle position, aux environs de Rouvres, où il était prêt à tirer à 8 heures du soir, à la demande des Polonais ${ }^{19}$. Déjà, ces derniers commençaient à apprécier l'apport précieux des canonniers du Régiment, si l'on en juge par cette inscription du Journal de guerre du Régiment:

Je tiens à souligner ici [c'est l'officier du service des renseignements qui écrit] ${ }^{20}$ l'excellente impression que les Polonais ont du Régiment et des officiers suivants qui les ont aidés dans leur avance: le major Tremblay, le capitaine Chabot, le major Lahaie, le capitaine Mercier, le capitaine Giroux et le lieutenant Caron ${ }^{21}$.

Ces deux derniers étaient alors à l'avant avec les Polonais.

A minuit, le 16 août, la Luftwaffe bombardait pendant vingt minutes les positions du Régiment. En même temps cir-

17 Schulman, op. cit., 189-190.

18 Herval, op. cit., 385.

19 Journal de guerre du Régiment, XXIX: 9.

20 Le Journal de guerre du Régiment fut d'abord tenu par le capitaine Jacques Dupuis, avocat, frère de Paul Dupuis, artiste bien connu de la TV; ensuite par le lieutenant Philippe Malouin, aujourd'hui lieutenant-colonel et notaire à Valleyfield.

21 Ibid. 
culait la rumeur que des parachutistes allemands s'apprêtaient à sauter dans les environs. Aussitôt, l'adjudant téléphonait à tous les P.C. du Régiment pour les aviser froidement que chacun devait défendre son poste l'arme à la main. Entre-temps, un prisonnier était amené au Q.G. régimentaire par l'aide médical du capitaine Brosseau. Ce prisonnier, en réalité, n'était pas un Allemand, mais un Russe enrôlé de force dans la Wehrmacht, qui s'était réfugié dans une tranchée à l'approche des bombardiers allemands. L'aide du capitaine Brosseau, réfugié dans la même tranchée, croyant avoir affaire à un confrère d'armes, lui offrit une cigarette; la lueur de l'allumette lui révéla à sa grande stupeur qu'il avait affaire à un uniforme gris-vert. Pris de peur, l'aide du capitaine Brosseau amena aussitôt le Russe épouvanté au Q.G. du Régiment, où il passa la nuit sous une table, près de l'adjudant, armé pour l'occasion de son revolver ${ }^{22}$.

La journée du 16 août se révélait plutôt calme au Régiment, alors que trois bataillons d'infanterie canadienne se battaient déjà dans Falaise ${ }^{23}$. Le lendemain matin, à 9 heures, le groupe de reconnaissance avançait vers Jort, afin de préparer une nouvelle position que le Régiment devait occuper dès 2 heures de l'après-midi. A l'entrée de ce village, - le premier non complètement évacué par la population civile, - des cadavres allemands encombraient encore la route. Ce même jour, on rapportait que l'avant-garde américaine, montant d'Alençon, ne se trouvait plus qu'à 4 milles de Trun, cette dernière localité se trouvant alors à portée des canons du Régiment. La route Falaise-Trun-Chambois devenait alors la cible de choix de toute l'artillerie alliée, étant la seule voie d'échappement possible, vers l'Est, des débris de la $7^{\mathrm{e}}$ armée allemande ${ }^{24}$. Malgré leur débâcle, les Allemands n'en réussissaient pas moins à déclencher, à 9 heures du soir, un violent tir de mortiers sur les positions du Régiment, au cours duquel deux canonniers de la $58^{\mathrm{e}}$ batterie étaient tués instantanément. Il s'agit du canonnier Bernier, fidèle ordonnance du capitaine Sévigny, et du canonnier Beau-

22 Ibid.

23 Ibid., 10.

24 Ibid., 11. 
lieu ${ }^{25}$. Tous deux étaient aussitôt inhumés par l'aumônier du Régiment près de la rivière de Jort. A $10 \mathrm{~h}$. $15 \mathrm{du}$ soir, la Luftwaffe venait relayer l'artillerie et réussissait cette fois à faire trois blessés dans la troupe $\mathrm{D}$, de la $58^{\mathrm{e}}$ batterie. On rapportait, à ce moment-là, que le couloir par lequel les Allemands pouvaient encore s'échapper, entre l'avant-garde polonaise, appuyée directement par le Régiment, et l'avant-garde américaine, appuyée par la division blindée du général Leclerc, était maintenant rétréci à 6 milles seulement ${ }^{26}$.

Le lendemain midi, 18 août, le Régiment avançait de nouveau, cette fois vers Marais-la-Chance, à trois milles au nordouest de Trun, afin de tirer sur les véhicules ennemis fuyant en désordre pour échapper à l'étau en voie de se refermer. De nouveau, cette journée-là, le Régiment était pilonné et mitraillé par l'aviation alliée, à tel point que, cette fois, le commandant décidait d'adresser une lettre de protestation auprès des autorités, leur faisant observer le résultat désastreux de telles erreurs sur le moral du Régiment ${ }^{27}$. Il faut dire que, dans le sillage des Polonais, le Régiment ne pouvait faire autrement que se trouver dans des localités beaucoup trop avancées pour sa sécurité, ce qui étonnait d'ailleurs les autres régiments d'artillerie, confortablement installés plus à l'arrière ${ }^{28}$.

Le 19 août, le major Tremblay était de nouveau avec les postes avancés polonais, où il parvenait à détruire au moins six Panzers, au grand ébahissement admiratif des Polonais, étouffant ainsi une contre-attaque ennemie.

Ce même jour, un officier du Régiment décrivait comme il suit les quelques jours précédents:

Je n'ai pas écrit depuis plusieurs jours parce que nous avons avancé souvent et que j'ai travaillé sans relâche. L'important, c'est que nous avancions. L'arrogante armée allemande de 1940 baisse enfin l'échine devant les coups mortels de la $1^{\text {re }}$ armée

25 Ibid.

26 Ibid.

27 Ibid., 12.

28 Ibid. 
canadienne. Nous pourrons dire que nous avons fait une grosse part dans l'annihilation de la $7^{\mathbf{e}}$ armée allemande encerclée depuis notre course vers Falaise... ${ }^{29}$.

\section{ACCUEIL DE LA POPULATION LIBEREE}

Pendant cette avance du Régiment avec la $1^{\text {re }}$ division blindée polonaise, l'accueil des Français libérés devenait de plus en plus délirant. "Les 'hurrah' fusent de partout au passage des Canadiens et des Polonais », écrivait un témoin normand à cette époque ${ }^{30}$. De fait, dans cette même lettre du 19 août, citée ci-dessus, voici ce qu'on peut lire à ce propos:

L'accueil des Français devient de plus en plus enthousiaste, à mesure que nous avançons. Cet aprèsmidi, en passant à travers un village avec ma jeep, j'ai dû ralentir pour écarter les femmes, les vieillards et les enfants qui voulaient me serrer la main et m'embrasser. Surtout lorsqu'ils nous entendent parler français, c'est du délire. Hier soir, M. le maire de l'endroit (s'il te plaît !) me donna une belle grande maison pour loger mes hommes. Aujourd'hui, je causais avec le directeur du Journal de Falaise. Il m'en a conté de bonnes sur les Boches: «Enfin, vous êtes venus, me disait-il. Nous vous attendions depuis cinq ans. Ah! si vous saviez ce qu'ils nous en ont donné du malheur ces salauds; et puis, les SS, pas de pitié, hein ! J'espère que vous allez les zigouiller ces cochons-là ! Ça viole les filles, ça pille tout ${ }^{31} \gg$.

Le 20 août, le Régiment se dirigeait encore vers une nouvelle position, à Moutiers-en-Auge, soit deux milles juste au nord de Trun. Décidément, le Régiment n'était guère très loin maintenant de l'extrême pointe de la pince destinée à sceller irrévocablement le sort de la $7^{\mathrm{e}}$ armée allemande.

\section{FIN DE LA BATAILLE DE NORMANDIE}

De fait, à compter de ce jour-là, la bataille de Normandie pouvait être considérée comme virtuellement terminée. Il est

${ }^{29}$ Archives de l'auteur, lettre du 19 août 1944.

30 Herval, op. cit., 392.

31 Archives de l'auteur, lettre du 19 août 1944. 
vrai que, la veille encore, les Polonais étaient encerclés et qu'ils n'avaient dû leur dégagement qu'au tir précis du Régiment, dirigé sans relâche et avec un courage héroïque par le capitaine Sévigny ${ }^{32}$. Mais dès l'aube du 20 , le groupe tactique du colonel de Langlade de la $2^{\text {e }}$ D.B. de Leclerc établissait le contact avec les Polonais ${ }^{33}$. Les Allemands qui avaient pu s'échapper de cette trappe fuyaient alors en toute hâte vers la Seine, alors que leurs malheureux confrères encerclés se faisaient hacher sans merci par l'artillerie, ou se rendaient par centaines à la fois. Cette bataille, décisive dans l'histoire de cette guerre, faisait écrire ce qui suit à un témoin normand:

Les régiments de la $1^{\text {re }}$ armée canadienne se sont brillamment distingués au cours de cette furieuse mêlée. Ils combattirent tous avec une égale bravoure. Ils firent preuve d'une splendide endurance et le nom des plus valeureux d'entre eux restera impérissable dans les annales de ce combat de géants que fut la bataille de Falaise, phase décisive de la bataille de France ${ }^{34}$.

Un autre témoin normand précisait davantage son admiration à l'égard des troupes canadiennes, en écrivant ce qui suit:

La reconnaissance des Falaisiens demeurera acquise aux héroïques unités alliées à qui revient l'honneur d'avoir chassé les Allemands de la Région: le Canadian Scottish Regiment, le Calgary Highlanders, le British Columbia Regiment, le Régiment de Maisonneuve, le South Saskatchewan Scottish, le Queen's Own Cameroon Highlanders, le Royal Highlanders, le Royal Hamilton, le Fusiliers Mont-Royal, le Régiment de la Chaudière, le $4^{e}$ Régiment canadien d'artillerie moyenne (canadien-français) et le $3^{e}$ Régiment canadien d'artillerie moyenne (canadienanglais) ${ }^{35}$.

Ce même jour, 20 août, un officier du Régiment exprimait par ces mots l'atmosphère de joie qui régnait alors dans le

32 Sévigny, op. cit., chap. 4.

33 Herval, op. cit., 432.

34 Ibid., 382.

35 Ibid., 383. 
Régiment: "Depuis quelques jours, la situation militaire est très brillante et le moral du Régiment est de plus en plus excellent ${ }^{36}{ }^{\text {}}$.

Dans une autre lettre, datée du même jour, il ajoutait:

Depuis notre arrivée en France, nous avons travaillé sept jours par semaine et 24 heures par jour sans arrêt. Mais nous récoltons aujourd'hui le fruit de notre effort soutenu: grâce à cette offensive ininterrompue, depuis Capiquet, Caen et Falaise, l'armée allemande est en déroute et la guerre achève. J'ai traversé toutes sortes d'expériences assez excitantes... mais une chose est certaine, c'est que je suis fier d'appartenir au $4^{\text {e }}$ Régiment. Nous avons accompli un travail étonnant... ${ }^{37}$.

\section{LA DEBÂCLE ALLEMANDE}

Pour ce qui est des Allemands, voici un autre extrait de lettre qui donne une idée plutôt amusante de leur retraite précipitée: «Un fermier me dit que les Boches ont filé si vite... que le capitaine allemand qui occupait ma chambre n'a pu enfiler sa culotte qu'en montant dans son camion. C'est la fuite effrénée ${ }^{38}$ ».

De fait, à partir de cette date, la retraite des débris de la $7^{\mathrm{e}}$ armée allemande prenait de plus en plus l'aspect d'une extraordinaire débandade. "... C'est une débâcle sans précédent, un stupéfiant amalgame de véhicules, de fantassins et de moteurs ${ }^{37}$ », «un 'Kolossal' charnier, le cimetière de l'armée von Kluge ... ${ }^{40}$ », "et cela dure jusqu'au 23 août, tandis que la canonnade se fait d'heure en heure plus distincte et plus proche ${ }^{41} \gg$. Voici comment un autre témoin normand décrivait cette scène: "Chars, piétons, camions, artillerie, chevaux, tout se rua vers la sortie, entre Falaise et Trun. Ce fut alors une

36 Archives de l'auteur, lettre du 20 août 1944.

37 Ibid., même date, à un autre correspondant.

38 Ibid., 21 août 1944.

29.

39 Charles Brisson, La ruche en flammes (1940-1944) (Elbeuf, 1950),

$40 \mathrm{Ibid}$.

41 Ibid. 
débâcle indescriptible, une déroute effroyable, un carnage horrible $^{42}$. 》

Pour finir, citons le témoignage le plus autorisé, celui du général Eisenhower lui-même, sur cette phase de la guerre, à laquelle avait si puissamment participé le Régiment:

Les canons alliés martelèrent le «terrain de mort 》 qui se réduisait toujours et alors que les unités de SS comme d'habitude se battaient jusqu'à l'extermination, l'infanterie ordinaire allemande se rendait à des effectifs toujours croissants ... La jolie région boisée à l'ouest d'Argentan était devenue le cimetière de l'armée qui, trois mois plus tôt, avait espéré avec confiance écraser l'invasion alliée sur les plages de Normandie ${ }^{43}$.

Cette défaite décisive de la Wehrmacht ne devait pas, malheureusement, marquer la fin prochaine de la guerre, comme on le croyait à l'époque. Toute cette furieuse bataille de Normandie n'a donc pas atteint le but qu'elle aurait dû atteindre, c'est-à-dire terminer la guerre dès 1944. Et pourtant, Dieu sait si elle avait coûté cher. En voici un bilan assez éloquent:

... que de ruines, que de misères, que de deuils ... ! Terrible bilan d'une bataille impitoyable, où chaque adversaire s'acharnant à détruire, détruisait en même temps les plus beaux monuments, les plus nobles paysages et jusqu'aux hameaux les plus paisibles, aux fermes les plus tranquilles ... Pâturages éventrés par les obus, ravinés par les chars, où pourrissent les cadavres des bêtes ... Forêts aux arbres décapités ... Et surtout la misère et le malheur des hommes, les milliers de morts sous les terribles bombardements ... Voici ce qui nous reste dans la mémoire de ce qui fut sans doute une des plus grandes batailles de l'histoire, la bataille de Normandie... ${ }^{44}$.

42 Herval, op. cit., 367.

43 Général Dwight Eisenhower, Les opérations en Europe du Corps expéditionnaire allié ( 6 juin 1944 au 8 mai 1945) (Paris, Berger-Levrault, 1947), 86.

44 Herval, op. cit., 10-11. 


\section{REPOS DU GUERRIER}

Le 22 août, le Régiment apprenait qu'il allait enfin bénéficier d'une période de repos. Il l'avait bien mérité. Le même jour, épuisé sans doute par l'effort incessant qu'il avait déployé depuis au-delà d'un mois, le lieut-col. Gagnon, commandant du Régiment, tombait malade de pleurésie et devait être hospitalisé pendant trois semaines. Aussitôt, le major Archer assumait le commandement du Régiment.

A 6 h. 30 du matin, le 23 août, le Régiment quittait sa dernière position de combat en Normandie pour se rendre à sa première position de repos, située à Bellou, au sud-est de Livarot. Le Q.G. régimentaire s'installait aussitôt dans le manoir de Bellou, immeuble admirablement bien conservé, et typique de la construction gentilhommière au XVI $\mathrm{XV}^{\mathrm{e}}$ siècle ${ }^{45}$. Évacué de la veille seulement par des officiers SS, ce magnifique manoir normand devait servir pendant quelques jours, - trop courts hélas ! - de lieu de repos bien mérité aux officiers du Régiment. La grande bataille terminée, chacun attendait avec optimisme la fin imminente de la guerre.$$
\text { (à suivre)* }
$$$$
\text { JACQUES GOUIN, }
$$$$
\text { ex-lieutenant d'artillerie, }
$$$$
\text { diplômé en sciences politiques (Ottawa), }
$$$$
\text { correspondant canadien à la Revue d'Histoire }
$$$$
\text { de la } 2^{\mathrm{e}} \text { guerre mondiale (France). }
$$

45 Jean de la Varende, Les Châteaux de Normandie, (Basse-Normandie) (Rouen, Henri Defontaine, 1937), 73.

* Trois autres articles sur le même sujet paraîtront, sous le titre * Artilleurs canadiens-français dans la libération du nord de la France, de la Belgique et de la Hollande ». 